\title{
Do Self-Control Preferences Help Explain the Puzzling Behavior of Asset Prices?
}

\author{
(revision of 2004-021 to be directed to R. King) \\ David N. DeJong and Marla Ripoll* \\ Department of Economics, University of Pittsburgh, Pittsburgh, PA 15260, USA
}

(Received February, 2004; Revised February, 2006)

\begin{abstract}
In the context of a simple asset-pricing environment, we study the ability of self-control preferences to account for the stock-price volatility, risk-free-rate and equity-premium puzzles. Using a full-information estimation procedure, we estimate the presence of a quantitatively small selfcontrol effect in the data. Moreover, with results obtained using CRRA preferences serving as a benchmark, we find that the adoption of self-control preferences makes only a marginal contribution towards a resolution of these puzzles.
\end{abstract}

Key Words: full-information estimation, risk-free rate, equity premium, stock price volatility

JEL Classification: E44; C11; C15

${ }^{*}$ Department of Economics, University of Pittsburgh, Pittsburgh, PA 15260, USA; Telephone: 412-648-2242; Fax: 412-648-1793; E-mails: dejong@pitt.edu, ripoll@pitt.edu.

This paper was previously circulated under the title "Self-Control Preferences and the Volatility of Stock Prices." For helpful comments we thank an anonymous referee, Jim Feigenbaum, Robert King, Per Krusell, Jean-Francois Richard, Tony Smith, Tom Tallarini, and seminar participants at the Institute for Advanced Studies (Vienna), and Charles, Comenius and Sabanci Universities. The usual disclaimer applies. 


\section{Introduction}

The theoretical characterization of asset-pricing behavior remains a challenging task. Three specific aspects of this behavior are particularly puzzling. First, stock prices are highly volatile relative to corresponding discounted dividend streams (LeRoy and Porter, 1981; Shiller, 1981; Grossman and Shiller, 1981). Also, average returns to holdings of relatively riskless assets (e.g., government bonds) are very low, while in turn, the average return premium generated by equity holdings over bond holdings is quite high (Mehra and Prescott, 1985; Weil, 1989). Despite the large literatures that have emerged in response to the original statements of these puzzles, definitive resolutions have yet to emerge (for surveys, see Kocherlakota, 1996; and Mehra and Prescott, 2003).

Recent work by Gul and Pesendorfer (2004) has suggested that the equity premium puzzle may arise from self-control problems faced by investors. Motivated by evidence indicating that experimental subjects willingly forgo future payoffs in return for relatively modest current rewards, Gul and Pesendorfer present an axiomatic characterization of this behavior, and illustrate its application in a variety of dynamic optimization problems. In one example, they illustrate the potential for the dynamic self-control (DSC) preferences they derive to intensify the equity premium. While their example is of limited empirical content (in particular, it is inconsistent with balanced-growth behavior evident in the data), below we show that these preferences do carry the potential under certain conditions to make headway (relative to a standard constant relative risk aversion (CRRA) preference specification) in explaining all three puzzles. However, the global empirical success of these preferences hinges on the relative strengths of competing effects, and boils down to an empirical question. The purpose of this paper is to shed light on this question.

Our analysis is based on two versions of a Lucas (1978) - type environment featuring a representative household. The versions differ only in their specification of preferences: results obtained using CRRA preferences serve as a benchmark, and are held in comparison with results obtained using a DSC specification. The model features two assets: equity, whose price reflects the household's valuation of the future dividend stream it is expected to generate; and a bond, whose price reflects the household's valuation of the sure claim to one unit of consumption it will generate in the next period. In equilibrium, consumption is financed by dividend payments and an exogenous endowment; innovations to these stochastic processes drive asset prices.

Our empirical analysis proceeds in two steps. We first estimate the parameters of each model; 
this is accomplished using the full-information Bayesian methodology of DeJong, Ingram and Whiteman (2000). We then assess the ability of each model to account for a collection of summary statistics that collectively enable us to quantify the volatility, risk-free-rate and equity premium puzzles. The data we employ are annual and span 1889 through 2004. They consist of equity prices and dividends corresponding to the S\&P's 500 index; prices of government bonds; and consumption of non-durables and services (details are provided in Section 4).

The results of our analysis are negative in two respects. First, we estimate the presence of a quantitatively small self-control effect in the data: temptation costs associated with adherence to long-term investment objectives are estimated to be on par with the utility loss associated with a permanent $0.1 \%$ reduction in steady state consumption. Second, relative to results obtained using the CRRA benchmark, we find that the adoption of self-control preferences makes only a marginal contribution towards a resolution of the three asset-pricing puzzles. ${ }^{2}$

There is a growing body of work that focuses on the potential role played by temptation and other self-control problems in influencing asset-pricing behavior. One study closely related to ours is that of Krusell, Kuruscu and Smith (2002), who explore the ability of a model featuring a small subset of agents who face a temptation to save to account for the equity premium puzzle. These agents end up dominating the equity market, thus their behavior determines asset prices. Calibrating their model to match the wealth distribution in the U.S., Krusell et al. find that they can account for the equity premium and low risk-free rate with a risk-aversion parameter in the neighborhood of 22. In addition, Huang, Liu and Zhu (2005) seek to identify the presence of a temptation effect using a panel data set constructed from the Consumer Expenditure Survey. Like us, the effect they identify turns out to yield little progress towards a resolution of the risk-free-rate and equity premium puzzles.

\section{The Model}

We consider a Lucas (1978)-type environment featuring a representative household situated in an endowment economy. There is a single consumption good in the economy, and two assets:

\footnotetext{
${ }^{2}$ These results contrast with those presented in an earlier version of this paper, in which we studied the role of temptation in accounting for the volatility puzzle in isolation. There we estimated a quantitatively significant temptation effect (equivalent to the utility loss associated with a $5.25 \%$ reduction in steady state consumption), and found that DSC preferences contributed modestly towards accounting for the volatility puzzle. Obviously, this finding is not robust once the broader range of asset-pricing behavior studied here is taken into account.
} 
equity shares, holdings of which at time- $(t-1)$ yield a per-share dividend payment $d_{t}$ at time $t$, and which carry a time- $t$ price in terms of the consumption good of $p_{t}$; and risk-free bonds, holdings of which at time- $(t-1)$ yield a per-unit claim to one sure unit of consumption at time $t$, and which carry a time- $t$ price in terms of the consumption good of $p_{t}^{f}$. Equity share holdings are given by $s_{t}$, bond holdings by $b_{t}$, the endowment received by the household by $e_{t}$, and consumption by $c_{t}$.

Household preferences follow the dynamic self-control specification proposed by Gul and Pesendorfer (2005), which encompass CRRA preferences as a special case. DSC preferences feature a temptation to liquidate all financial holdings in a given period; resisting this temptation imposes a self-control utility cost. Letting $s$ and $s^{\prime}$ denote time- $t$ and $(t+1)$ holdings of equity, etc., the household's problem can be formulated recursively as

$$
W(s, b, P)=\max _{s^{\prime}, b^{\prime}}\left\{u(c)+v(c)+\beta E W\left(s^{\prime}, b^{\prime}, P^{\prime}\right)\right\}-\max _{\widetilde{s}^{\prime}, \widetilde{b}^{\prime}} v(\widetilde{c}),
$$

where $P=\left(p, p^{f}, d, e\right), u(c)$ and $v(c)$ are Von Neuman-Morgenstern utility functions, the discount factor $0<\beta<1$, and $E$ is the conditional expectations operator (given information available at time $t$ ). Maximization is made subject to the following budget constraints:

$$
\begin{aligned}
& c=d s+b+e-p\left(s^{\prime}-s\right)-p^{f} b^{\prime}, \\
& \widetilde{c}=d s+b+e-p\left(\widetilde{s}^{\prime}-s\right)-p^{f} \widetilde{b}^{\prime} .
\end{aligned}
$$

The variables $\left(\widetilde{c}, \widetilde{s}^{\prime}, \widetilde{b}^{\prime}\right)$ are temptation counterparts to $\left(c, s^{\prime}, b^{\prime}\right)$, and the term $v(c)-\max v(\widetilde{c})$ $\leq 0$ represents the disutility of self-control the household suffers given the choice of $c$ instead of the temptation alternative $\widetilde{c}$.

Due to balanced-growth considerations discussed below, the functional forms for $u(c)$ and $v(c)$ employed in our empirical implementation are given by

$$
\begin{aligned}
& u(c)=\frac{c^{1-\gamma}}{1-\gamma}, \\
& v(c)=\lambda u(c) .
\end{aligned}
$$

Note that the parameter $\lambda$ is critical in determining the strength of potential self-control effects. In particular, preferences are of the standard CRRA type given $\lambda=0$, and ceteris paribus, the 
disutility of self-control intensifies as $\lambda$ increases.

Note that the optimal choices of $\widetilde{s}$ and $\widetilde{b^{\prime}}$ are zero: in each period, the household is tempted to liquidate its assets and equate temptation consumption $\widetilde{c}$ to all available wealth. That is, $\widetilde{c}=d s+b+e+p s$, reducing the household's problem to

$$
W(s, b, P)=\max _{s^{\prime}, b^{\prime}}\left\{u(c)+v(c)+\beta E W\left(s^{\prime}, b^{\prime}, P^{\prime}\right)\right\}-v(d s+b+e+p s),
$$

subject to (2). First-order conditions associated with the choices of $s^{\prime}$ and $b^{\prime}$ are given by

$$
\left[u^{\prime}(c)+v^{\prime}(c)\right] p=\beta E\left[\left(u^{\prime}\left(c^{\prime}\right)+v^{\prime}\left(c^{\prime}\right)-v^{\prime}\left(d^{\prime} s^{\prime}+b^{\prime}+e^{\prime}+p^{\prime} s^{\prime}\right)\right)\left(d^{\prime}+p^{\prime}\right)\right]
$$

and

$$
\left[u^{\prime}(c)+v^{\prime}(c)\right] p^{f}=\beta E\left[u^{\prime}\left(c^{\prime}\right)+v^{\prime}\left(c^{\prime}\right)-v^{\prime}\left(d^{\prime} s^{\prime}+b^{\prime}+e^{\prime}+p^{\prime} s^{\prime}\right)\right]
$$

Since households are identical, trading will not take place in equilibrium, nor will lending or borrowing. Normalizing share holdings to unity, and with $b=b^{\prime}=0$, in equilibrium

$$
c=d+e
$$

Combining (6) with the specification $v(c)=\lambda u(c)$, the first-order conditions yield the following equilibrium prices:

$$
\begin{gathered}
p=\beta E\left[M R S \cdot\left(d^{\prime}+p^{\prime}\right)\right], \\
p^{f}=\beta E(M R S),
\end{gathered}
$$

where the term $M R S$ corresponds to the household's intertemporal marginal rate of substitution between $t$ and $t+1$ as given by

$$
M R S=\frac{u^{\prime}\left(d^{\prime}+e^{\prime}\right)+\lambda\left[u^{\prime}\left(d^{\prime}+e^{\prime}\right)-u^{\prime}\left(d^{\prime}+e^{\prime}+p^{\prime}\right)\right]}{(1+\lambda) u^{\prime}(d+e)} .
$$

Note once again that when $\lambda=0$, self-control effects disappear and equilibrium prices associated with CRRA preferences emerge.

In order to develop some intuition on the differences between the $M R S$ in the self-control and 
CRRA cases, notice first that the denominator of the $M R S$ corresponds to the marginal utility cost of saving today. For given $d$ and $e$, this term is increasing in $\lambda$. This larger marginal utility cost of saving today is a direct consequence of the presence of temptation, whose strength is measured by $\lambda$.

The numerator of the $M R S$ is the marginal utility benefit realized tomorrow from increased savings today. Note that the additional term (relative to the CRRA case) $\lambda\left[u^{\prime}\left(d^{\prime}+e^{\prime}\right)-u^{\prime}\left(d^{\prime}+e^{\prime}+p^{\prime}\right)\right]$ represents the marginal impact of an increase in wealth on the utility cost of self-control. Since $u($.$) is concave, this additional term is positive, indicating that an increase in wealth reduces the$ utility cost of self-control. ${ }^{3}$ Thus, given our specification of the temptation function $v(c)=\lambda u(c)$, the numerator of the $M R S$ is larger than in the CRRA case.

To summarize, the discussion above suggests that under self-control preferences, households experience two forces. On one hand, they experience a temptation to consume all their wealth, which increases the marginal utility cost of saving today, and tends to make the $M R S$ lower than in the CRRA case. On the other hand, they experience self-control costs; these costs are decreasing in wealth given the concavity of the temptation function. This force increases the future marginal utility of saving today, and tends to make the $M R S$ higher than in the CRRA case.

The model is closed by augmenting (6), (7) and (8) with the specification of stochastic processes for $\left(d_{t}, e_{t}\right)$. These are given by

$$
\begin{aligned}
& \ln d_{t}=\left(1-\rho_{d}\right) \ln \bar{d}_{t}+\rho_{d} \ln d_{t-1}+\varepsilon_{d t} \\
& \ln e_{t}=\left(1-\rho_{e}\right) \ln \bar{e}_{t}+\rho_{e} \ln e_{t-1}+\varepsilon_{e t}
\end{aligned}
$$

with

$$
\left[\begin{array}{l}
\varepsilon_{d t} \\
\varepsilon_{e t}
\end{array}\right] \sim \text { iid } N(0, \Sigma) .
$$

Regarding notation, bars are used to denote steady state values. Note that time subscripts are included in the specifications of $\left(\bar{d}_{t}, \bar{e}_{t}\right)$; this is done in order to introduce growth into the model. The reason for this is that empirically, $\left(p_{t}, d_{t}, c_{t}\right)$ exhibit roughly balanced-growth trajectories (while $p_{t}^{f}$ exhibits stationary fluctuations about a constant mean). We therefore introduce growth

\footnotetext{
${ }^{3}$ For motivation, think of an Atkins dieter facing a dessert cart: she will suffer less from skipping dessert having just polished off a $12-\mathrm{oz}$ T-bone rather than a 4 -oz tofu patty.
} 
into the model in order to align theoretical variables with their empirical counterparts (details regarding alignment are provided in Section 4).

We characterize growth behavior following Shiller (1981), whose analysis of the volatility puzzle was based on the assumption that dividends and prices are trend-stationary; and DeJong (1992) and DeJong and Whiteman (1991, 1994), who provided subsequent empirical support for this assumption. In order to capture trend-stationarity and balanced growth, we specify $\left(\bar{d}_{t}, \bar{e}_{t}\right)$ as

$$
\begin{aligned}
& \ln \bar{d}_{t}=\phi_{0}^{d}+\phi_{1} t \\
& \ln \bar{e}_{t}=\phi_{0}^{e}+\phi_{1} t .
\end{aligned}
$$

Since $c_{t}=d_{t}+e_{t},(10)$ characterizes $\left(d_{t}, e_{t}, c_{t}\right)$ as following balanced-growth paths with an annual average growth rate of $\phi_{1}$. Moreover, with $p_{t}$ and $p_{t}^{f}$ obeying the pricing functions (7) and (8), the functional forms (4) and (5) chosen for $u(c)$ and $v(c)$ guarantee the existence of a balanced growth path under which $p_{t}$ grows at the annual average rate of $\phi_{1}$, and $p_{t}^{f}$ will exhibit stationary fluctuations around a constant steady state value. ${ }^{4}$

\section{Asset Pricing Puzzles}

Here we provide intuition regarding the potential for self-control preferences to make progress towards a resolution of the volatility, risk-free-rate and equity-premium puzzles. Beginning with the former, it is useful to rewrite the equity pricing equation (7) as

$$
p=\beta E\left[\left(\frac{\left(d^{\prime}+e^{\prime}\right)^{-\gamma}+\lambda\left[\left(d^{\prime}+e^{\prime}\right)^{-\gamma}-\left(d^{\prime}+e^{\prime}+p^{\prime}\right)^{-\gamma}\right]}{(1+\lambda)(d+e)^{-\gamma}}\right)\left(d^{\prime}+p^{\prime}\right)\right]
$$

and to consider the response of $p$ to an increase in $e$ or $d$. In the CRRA case $(\lambda=0)$, households pursue a standard intertemporal "smoothing" motive: they seek to shift a portion of the resulting increase in wealth to a future period (in which the expected marginal utility of consumption is greater). This smoothing motive, coupled with an accompanying expected increase in the value of equity in the next period, generates a corresponding increase in $p$.

Two facets of the data are difficult to characterize via this smoothing mechanism: stock prices

\footnotetext{
${ }^{4}$ We thank an anonymous referee for emphasizing the importance of the balanced-growth restrictions embodied in (4) and (5).
} 
are closely correlated with dividends, but are far more volatile. Note that the volatility of $p$ can be matched if $e$ were the predominant source of innovations to $p$; but if this were the case, the correlation between $p$ and $d$ would be weakened. By intensifying the household's smoothing motive, increases in the coefficient of relative risk aversion parameter $\gamma$ can also help generate increased volatility in $p$. But as noted originally by Grossman and Shiller (1981), the required specification of $\gamma$ must implausibly high to successfully account for the degree of volatility observed in the data.

Although it is difficult to establish analytically whether self-control preferences can amplify the volatility of $p$, we can at least identify one effect that works in this direction. This effect pertains to the marginal utility benefit realized tomorrow from increased savings today:

$$
\left(d^{\prime}+e^{\prime}\right)^{-\gamma}+\lambda\left[\left(d^{\prime}+e^{\prime}\right)^{-\gamma}-\left(d^{\prime}+e^{\prime}+p^{\prime}\right)^{-\gamma}\right] .
$$

As discussed above, with $v(c)$ concave, the additional term (relative to the CRRA case)

$$
\lambda\left[\left(d^{\prime}+e^{\prime}\right)^{-\gamma}-\left(d^{\prime}+e^{\prime}+p^{\prime}\right)^{-\gamma}\right]>0
$$

indicating that an increase in wealth reduces the utility cost of self-control. This effect serves to amplify the response of $p$ to an innovation to either $d$ or $e$, for a given value of $\gamma$.

Turning to the risk-free-rate, the puzzle here is that consumption growth is relatively high (indicating a relatively high willingness to save on the part of households), despite the fact that households seem to have a strong consumption-smoothing motive, and that the return to riskfree savings is low. To evaluate the potential for self-control preferences to help account for these observations, it is useful to define $R^{f}$ to be the gross real risk-free return, and re-write (8) as

$$
\frac{1}{R^{f}}=\beta E(M R S)=\beta E\left[\frac{\left(d^{\prime}+e^{\prime}\right)^{-\gamma}}{(d+e)^{-\gamma}}-\frac{\lambda}{(1+\lambda)} \frac{\left(d^{\prime}+e^{\prime}+p^{\prime}\right)^{-\gamma}}{(d+e)^{-\gamma}}\right]
$$

For given $d, e, \beta$ and $\gamma$, we can use this equation to compare the implied average risk-free return for the CRRA case and under self-control preferences. Notice that the first term in the square brackets is the $M R S$ under CRRA preferences, while the second term captures additional effects due to the presence of temptation and self-control costs. In order to establish whether the risk-free return is increasing or decreasing in $\lambda$, notice that there are two types of additional effects. 
First, there is a direct effect operating through $\lambda /(1+\lambda)$, which makes $R^{f}$ increasing in $\lambda$. In other words, due to this effect the risk-free rate should be larger given self-control preferences: the return to savings must be larger to prevent households from succumbing to temptation. The low observed risk-free rate is even more puzzling in light of this effect. However, the second additional effect may work in the opposite direction. This second effect corresponds to the indirect influence of $\lambda$ on $R^{f}$ through the future price of the risky asset $p^{\prime}$. For instance, if $p$ is increasing in $\lambda$, then the second effect may offset the first and make $R^{f}$ decreasing in $\lambda$. But this cannot be established analytically. The potential contribution of self-control effects in accounting for the risk-free rate puzzle is therefore an empirical question.

Finally, the equity premium puzzle refers to the large gap in the average returns generated by investing in equity rather than relatively riskless assets. Given observed differences in the inherent risk associated with investing in these assets, households must be implausibly risk-averse to account for the observed gap in returns.

To evaluate the potential for self-control preferences to help account for this puzzle, let $R$ denote the expected gross return on the risky asset. Then the equilibrium price of the risky asset implies

$$
\beta E[M R S \cdot R]=1
$$

so that

$$
E(R)=\frac{1-\beta \operatorname{cov}(M R S, R)}{\beta E(M R S)}
$$

Thus the expected return on the risky asset is a function of both $E(M R S)$ and the covariance between the $M R S$ and $R$. As with the risk-free rate, note that the expected return on the risky asset is an inverse function of $E(M R S)$, which as discussed above may be larger or smaller under DSC preferences than under CRRA preferences. The presence of $\operatorname{cov}(M R S, R)$ in this case adds an additional source of ambiguity, as an analytical characterization of this term is unavailable.

Lacking an analytical characterization, we sketch a stylized example following a similar exercise conducted by Gul and Pesendorfer (2004). In their example, they show that if $u(c)+v(c)$ is concave and $v(c)$ is convex, self-control preferences are unambiguously consistent with a larger equity premium than one would expect under CRRA preferences. However, the convexity of $v(c)$ is incompatible with the balanced-growth behavior of $(c, d, p)$ characterized above, thus we reconduct 
their example by characterizing $v(c)$ as in $(5)$.

For purposes of tractability, consider a simplification of the model under which $e_{t}$ is eliminated and $\left\{d_{t}\right\}$ is serially uncorrelated with mean 1 and variance $\sigma^{2}$. Also for tractability, fix $\gamma=1$, so that $u(c)=\ln (c)$ and $v(c)=\lambda \ln (c)$. Following Gul and Pesendorfer's (2004) notation, let $D$ denote the realized value of $d$ in the current period. Notice that the price of the risky asset depends on the dividend realization as described by the function $p(d)$. The equity pricing equation in this example is given by

$$
p(D)=\beta E\left[\frac{(1+\lambda) / d-\lambda /(p(d)+d)}{(1+\lambda) / D}(p(d)+d)\right] .
$$

Under the assumptions of the example, the expected value of asset prices will be a constant $p$, thus we can write

$$
p=\frac{\beta D}{(1+\lambda)[1-\beta D E(1 / d)]} .
$$

Since the expected gross return on the risky asset $R$ is given by

$$
R=E\left[\frac{d+p}{p}\right]=\frac{1+p}{p}
$$

it follows that

$$
\frac{\partial R}{\partial \lambda}=\frac{-1}{p^{2}} \frac{\partial p}{\partial \lambda}=\frac{1}{p(1+\lambda)}>0
$$

which implies that in this special example, as the strength of the temptation $\lambda$ increases, the expected return on the risky asset unambiguously increases.

Next, the associated equilibrium equation for risk-free returns is given by

$$
\frac{1}{R^{f}} \equiv p^{f}=\beta E\left[\frac{(1+\lambda) / d-\lambda /(p(d)+d)}{(1+\lambda) / D}\right]
$$

and thus

$$
\frac{\partial\left(1 / R^{f}\right)}{\partial \lambda}=-\frac{1}{(1+\lambda)^{2}} \beta D E[1 /(p(d)+d)]+\frac{\beta \lambda D}{(1+\lambda)} E\left[1 /(p(d)+d)^{2}\right] \frac{\partial p}{\partial \lambda}<0
$$

where the first term corresponds to the direct effect of $\lambda$ on $1 / R^{f}$, while the second one represents the indirect effect of $\lambda$ on $1 / R^{f}$ through the risky asset price $p$. The equation above implies that in this special example, as the strength of the temptation $\lambda$ increases, the expected return on the 
risk-free bond unambiguously increases. Finally, since the equity premium is given by $R-R^{f}$, but in our example both $R$ and $R^{f}$ are increasing in $\lambda$, we cannot easily establish whether the equity premium will be increasing or decreasing in $\lambda$. Thus when $v(c)$ is taken as concave rather than convex, the potential contribution of self-control effects in accounting for the equity-premium puzzle is once again an empirical question. We now turn to an investigation of this question.

\section{Results}

\subsection{Data Description}

The data we consider are updated versions of those studied, e.g., by Shiller (1981) and Mehra and Prescott (1985). The data are annual, and span 1889 through 2004. Equity prices are represented by the S\&P's 500 monthly composite stock price index for January, divided by the producer price index (January PPI starting in 1900, annual average PPI from 1889-1899); dividends are represented by total dividends for the calendar year accruing to the portfolio represented by the stocks in the S\&P 500 index, divided by the average PPI for the year. Risk-free prices are represented as the inverse of the risk-free rate, computed as nominal government bond yields deflated by the annual consumer price index (CPI). Finally, consumption is real per captia consumption of non-durables and services, where again deflation is accomplished using the CPI. ${ }^{5}$

Along their balanced-growth path, consumption, equity prices and dividends grow at an estimated annual average rate of $1.58 \%$ (the growth-rate parameter $g$ is fixed at this value in the estimation stage). In contrast, risk-free prices fluctuate about a fixed sample mean of 0.985 . The sample mean (standard deviation) return to equity is $7.98 \%$ (18.30\%), compared with $1.84 \%$ (5.59\%) for the risk-free asset. The corresponding equity premium is $6.14 \%$ (17.91\%), which is similar to the premium of $6.18 \%(16.67 \%)$ reflected in the data set studied by Mehra and Prescott (which spanned 1889-1978).

Asset prices and dividends are illustrated in Figure 1. Equity prices and dividends are depicted as logged deviations about their balanced-growth paths, and risk-free prices are depicted as logged deviations about their sample average. Equity prices are positively correlated with dividends (0.44), but are far more volatile (the ratio of their standard deviations is 1.55). In contrast, risk-free prices

${ }^{5}$ The data are available for downloading from Robert Shiller's website:
http://www.econ.yale.edu/ ${ }^{\sim}$ shiller/data/chapt26.xls


move relatively independently and smoothly: correlations with equity prices and dividends are less than 0.07 ; and the ratio of its standard deviation relative to equity prices is 0.13 .

\subsection{Data Alignment}

To facilitate model approximation and estimation, we must induce stationarity in the model variables and their empirical counterparts. Regarding the model variables, we introduce the normalization $c_{t}^{*}=c_{t} /(1+g)^{t}$ for $\left(c_{t}, d_{t}, e_{t}, p_{t}\right)$, with $g$ denoting the common growth rate of these variables. With this normalization, the model characterizes $y_{t}=\left(c_{t}^{*}, d_{t}^{*}, e_{t}^{*}, p_{t}^{*}, p_{t}^{f}\right)^{\prime}$ as obeying

$$
\begin{gathered}
c_{t}^{*}=d_{t}^{*}+e_{t}^{*} \\
p_{t}^{f}=(1+g)^{-\gamma} \beta E\left[\frac{(1+\lambda)\left(d_{t+1}^{*}+e_{t+1}^{*}\right)^{-\gamma}-\lambda\left(d_{t+1}^{*}+e_{t+1}^{*}+p_{t+1}^{*}\right)^{-\gamma}}{(1+\lambda)\left(d_{t}^{*}+e_{t}^{*}\right)^{-\gamma}}\right] \\
p_{t}^{*}=(1+g)^{1+\gamma} \beta\left(d_{t+1}^{*}+p_{t+1}^{*}\right) E\left[\frac{(1+\lambda)\left(d_{t+1}^{*}+e_{t+1}^{*}\right)^{-\gamma}-\lambda\left(d_{t+1}^{*}+e_{t+1}^{*}+p_{t+1}^{*}\right)^{-\gamma}}{(1+\lambda)\left(d_{t}^{*}+e_{t}^{*}\right)^{-\gamma}}\right] \\
\ln d_{t}^{*}=\rho_{d} \ln d_{t-1}^{*}+\varepsilon_{d t} \\
\ln e_{t}^{*}=\rho_{e} \ln e_{t-1}^{*}+\varepsilon_{e t} .
\end{gathered}
$$

The elements of $y_{t}$ exhibit stationary fluctuations about their constant steady state values $\left(\bar{c}, \bar{d}, \bar{e}, \bar{p}, \bar{p}^{f}\right)$; the persistence of these fluctuations is determined by $\left(\rho_{d}, \rho_{e}\right)$.

Our empirical representation of the model is based on a log-linear approximation of (11) - (15), which is written in terms of the normalized variables $y_{t}$ recast in terms of logged deviations from steady state values. Let these logged deviations be given by

$$
x_{t}=\left(\ln \left(c_{t}^{*} / \bar{c}\right), \ln \left(d_{t}^{*} / \bar{d}\right), \ln \left(e_{t}^{*} / \bar{e}\right), \ln \left(p_{t}^{*} / \bar{p}\right), \ln \left(p_{t}^{f} / \bar{p}^{f}\right)\right)^{\prime}
$$

In order to produce empirical counterparts to the observable elements of $x_{t}$ (in our case $c, d$, $p$, and $\left.p^{f}\right)$, we proceed in four steps. First, we calculate steady state values $\left(\bar{c}, \bar{d}, \bar{e}, \bar{p}, \bar{p}^{f}\right)$ for a given parameterization of the model. Second, we estimate log-linear trajectories for $(c, d, p)$ subject to two restrictions: the trajectories feature a common growth rate; and the intercepts adhere to steady state ratios implied by the model. Third, we obtain de-meaned and de-trended versions of $(c, d, p)$ as the difference between their logged levels and their estimated log-linear 
trajectories. Fourth, we obtain a de-meaned version of $p^{f}$ as the difference between its logged level and its logged steady state value. Note that these steps are repeated for each candidate model parameterization we consider, since steady state values are functions of the parameters. Hereafter, the de-meaned/de-trended empirical counterparts to the observable elements of $x_{t}$ are denoted as

$$
X_{t}=\left(\ln \left(p_{t} / \overline{p_{t}}\right), \ln \left(d_{t} / \overline{d_{t}}\right), \ln \left(p_{t}^{f} /{\overline{p_{t}}}^{f}\right), \ln \left(c_{t} / \overline{c_{t}}\right)\right)^{\prime}
$$

where $\bar{p}_{t}$, etc. represent estimates of deterministic behavior.

This method of inducing stationarity serves two purposes. First, it ensures that the processed data are in the precise form in which they are characterized in the model. Second, by aligning intercepts (or means in the case of $p^{f}$ ) with corresponding steady state values, we preserve information conveyed by the levels of the data in helping determine the parameterization of the model. For example, note from (12) that the steady state value of $p^{f}$ under CRRA preferences is given by $\beta(1+g)^{-\gamma}$. Specifications of $\beta$ and $\gamma$ that fail to closely align $\bar{p}^{f}$ with the sample mean of $p^{f}$ will be penalized heavily in the estimation stage; or in other words, the sample mean of $p^{f}$ provides a strong source of identification in estimating these parameters under this approach to inducing stationarity.

\subsection{Estimation}

Estimation is accomplished using the full-information Bayesian procedure developed by DeJong, Ingram and Whiteman (2000). Briefly, this works as follows. Using a first-order Taylor-series expansion, we obtain a log-linear approximation of the model (represented in (11) - (15)), the solution of which takes the form of the state system

$$
\begin{aligned}
& x_{t}=F(\mu) x_{t-1}+v_{t}, \\
& v_{t}=G \varepsilon_{t}, \quad \varepsilon_{t}=\left(\varepsilon_{d t}, \varepsilon_{e t}\right)^{\prime},
\end{aligned}
$$

where $\mu$ is the collection of model parameters. The model variables $x_{t}$ map into the observables $X_{t}$ via the observation equation

$$
X_{t}=H^{\prime} x_{t}+u_{t}, \quad E u_{t} u_{t}^{\prime}=\Sigma_{u}
$$


where $u_{t}$ is a vector of serially uncorrelated measurement errors associated with the observation of $X_{t}$. These errors are introduced because there are more observable variables than there are sources of uncertainty in the model. With fewer sources of uncertainty than observables, various linear combinations of variables will be predicted to be deterministic, and departures from this prediction will render likelihood analysis untenable (for elaboration on this so-called stochastic singularity issue, see Ingram, Kocherlakota and Savin, 1994). We restrict these errors to be uncorrelated across equations, so that $\Sigma_{u}$ is diagonal.

Let $X=\left\{X_{t}\right\}_{t=1}^{T}$. Coupled with the assumption of normality for $\varepsilon_{t}$, (16) - (18) yield a likelihood function $L(X \mid \mu)$ that can be evaluated using the Kalman filter. Coupling $L(X \mid \mu)$ with a prior distribution $\pi(\mu)$ yields a posterior distribution $P(\mu \mid X) \propto \pi(\mu) L(X \mid \mu)$. This distribution, along with corresponding distributions over functions $g(\mu)$, may be approximated using numerical integration methods.

In specifying $\pi(\mu)$, our goal is to place an emphasis on standard ranges of parameter values, while assigning sufficient prior uncertainty to allow the data to have a nontrivial influence on our estimates. The priors we use for this purpose are summarized in Table 1. In all cases, prior correlations across parameters are zero; and each of the informative priors we specify is normally distributed (but truncated when appropriate).

The prior mean (standard deviation) of the discount factor $\beta$ is 0.96 (0.02), implying an annual discount rate of $4 \%$. The prior for the risk aversion parameter $\gamma$ is centered at 2 (1). We specified the usual non-informative prior over the covariance matrices $\Sigma$ and $\Sigma_{u}$ (proportional, e.g., to $\operatorname{det}(\Sigma)^{-(m+1) / 2}$, where $m$ is the dimension of $\left.\Sigma\right)$, and centered the priors over the persistence parameters $\left(\rho_{d}, \rho_{e}\right)$ at $0.85(0.025)$. The priors over $\left(\beta, \rho_{d}, \rho_{e}\right)$ are truncated from below at zero and from above at 0.995 (in order to remain in a stable region of the parameter space). Finally, we specified a normal distribution for $\lambda$ centered and truncated from below at 0 (0.1). Given truncation, the mean of this distribution is $0.0796(0.06)$. Regarding this specification, as $\lambda$ ranges from 0 to 0.1, implied temptation costs associated with adherence to long-term investment objectives are on par with the utility loss associated with a permanent reduction in steady state consumption in the range of $0 \%$ to $15 \%$ (holding the additional parameters fixed at their prior means). 


\subsection{Results}

Parameter estimates are presented in Table 1. Without exception, estimates obtained under both CRRA and DSC preferences lie within one posterior standard deviation of each other, providing the first indication that the replacement of CRRA with DSC preferences matters little empirically. The marginal posterior distribution of $\beta$ turns out to be a spike at its upper bound of 0.995, which is a manifestation of the risk-free rate puzzle: the low average return to the riskless asset reflects minimal discounting on the part of the household. Regarding $\gamma$, recall that larger values help account for the volatility and equity-premium puzzles, but make the low average riskfree rate harder to understand. Empirically, the former effects outweigh the latter. The posterior distribution of $\gamma$ shifts well to the right and is tightly distributed relative to our prior: posterior means (standard deviations) are in the neighborhood of $3.5(0.1)$. Distributions over the persis-

tence parameters $\left(\rho_{d}, \rho_{e}\right)$ are also rightshifted relative to our prior, in all cases centered tightly in the neighborhood of 0.92. Finally, regarding the estimates obtained for $\Sigma$ and $\Sigma_{u}$, note that the standard deviations of the measurement errors associated with the observation of prices and dividends in (18) have distributions spiked at 0 . This is due to the fact that with two sources of model uncertainty and four observable variables, only two additional sources of error are required to eliminate stochastic singularity.

Regarding $\lambda$, note that the posterior mean and standard deviation estimates reported in Table 1 closely mirror their prior counterparts. The reason for this is illustrated in Figure 2. The top panel of the figure depicts the behavior of the log-likelihood function as $\lambda$ ranges away from 0 . This behavior is depicted by fixing the additional parameters at their posterior means. Note that the likelihood function exhibits a slight preference for a specification of $\lambda$ in the neighborhood of 0.003 , implying a modest temptation cost on par with the utility loss associated with a permanent $0.1 \%$ reduction in steady state consumption (bottom panel).

This weak evidence of a temptation effect is obtained despite the fact that temptation carries with it the unambiguous potential to help account for the stock-price volatility puzzle. Recall that this potential was manifested in an earlier version of this paper, in which we studied the volatility puzzle in isolation. That this weak evidence is obtained given the broader range of asset-pricing behavior considered here indicates that temptation is not helpful in accounting for the risk-free rate and equity-premium puzzles. 
Further support for this conclusion is presented in Figures 3 and 4. Four sets of marginal posterior distributions over summary statistics are depicted in each figure; distributions obtained using CRRA (DSC) preferences are depicted in Figure 3 (4). Collectively, the statistics are designed to convey the performance of each model in accounting for the volatility, risk-free-rate and equitypremium puzzles.

The first statistic is the difference in ratios of the standard deviations of prices and dividends implied by a given parameterization of the model $(M)$ and the ratio observed in the data $(D)$ :

$$
\frac{\sigma(p)}{\sigma(d)}_{M}-\frac{\sigma(p)}{\sigma(d)}_{D}
$$

The ratio predicted by the model is of course a function of its parameterization; integration over the posterior distribution of the model parameters yields the posterior distribution over the statistic depicted in the figures. The second statistic is the difference in the correlation between prices and dividends predicted by the model and observed in the data:

$$
\operatorname{corr}(p, d)_{M}-\operatorname{corr}(p, d)_{D}
$$

Recall that the empirical challenge associated with the volatility puzzle is to capture the relatively high volatility of prices while preserving the correlation pattern observed between prices and dividends.

The third and fourth statistics were used by Kocherlakota (1996) to summarize the risk-free-rate and equity-premium puzzles. Regarding the risk-free rate, recalling that $1 / r^{f}=R^{f}$ and $c=d+e$, the pricing kernel for $p^{f}$ in (8) may be arranged as

$$
\beta E\left[\frac{(1+\lambda) u^{\prime}\left(c_{t+1}\right)-\lambda u^{\prime}\left(c_{t+1}+p_{t+1}\right)}{(1+\lambda) u^{\prime}\left(c_{t}\right)}\right] R_{t}^{f}-1=0
$$

Rearranging (7) in similar fashion yields

$$
\beta E\left[\frac{(1+\lambda) u^{\prime}\left(c_{t+1}\right)-\lambda u^{\prime}\left(c_{t+1}+p_{t+1}\right)}{(1+\lambda) u^{\prime}\left(c_{t}\right)}\right] R_{t}-1=0
$$

where $R=\left(p^{\prime}+d^{\prime}\right) / p$. Kocherlakota's risk-free rate statistic is obtained by approximating the population means in (19) with a sample average; and his equity premium statistic is obtained by 
using a sample average to approximate the population means obtained by subtracting the lefthand-sides of (19) and (20):

$$
E\left[\frac{(1+\lambda) u^{\prime}\left(c_{t+1}\right)-\lambda u^{\prime}\left(c_{t+1}+p_{t+1}\right)}{(1+\lambda) u^{\prime}\left(c_{t}\right)}\right]\left(R_{t}-R_{t}^{f}\right)=0
$$

For all four statistics, empirical success is associated with calculated values of zero.

Figure 3 presents a benchmark illustration of the three puzzles. The posterior mean (standard deviation) of the distribution over the $\sigma(p) / \sigma(d)$ statistic is $-0.437(0.199)$, and the bulk of the distribution lies below zero: the predicted volatility of prices falls short of its empirical benchmark. In turn, the risk-free-rate statistic is tightly centered around -0.0494, and the equity-premium statistic is tightly centered around 0.0565. (In comparison, the statistics obtained by Kocherlakota using $\beta=0.99, \gamma=3.5$ and a data set spanning 1889-1978 are -0.051 and 0.048.)

Figure 4 presents analogous distributions obtained using the DSC specification. The distributions obtained for the risk-free-rate and equity-premium statistics are virtually identical to their CRRA counterparts, while the distributions over $\sigma(p) / \sigma(d)$ and $\operatorname{corr}(p, d)$ are shifted slightly towards zero. In particular, the posterior mean (standard deviation) over $\sigma(p) / \sigma(d)$ is $-0.424(0.217)$ in this case: an improvement, but only a minimal one.

\section{Conclusion}

We have studied the ability of the dynamic self-control preferences proposed by Gul and Pesendorfer (2004) to account for the stock-price volatility, risk-free-rate and equity-premium puzzles. Applying a full-information estimation procedure to an updated version of Mehra and Prescott's (1985) data set, we estimate the presence of a quantitatively small self-control effect in the data,

as well as a marginal contribution of self-control preferences in explaining the puzzling behavior of asset prices.

Our results run contrary to the initial optimism that followed Gul and Pesendorfer's (2004) proposal that dynamic self-control preferences carry the potential to help account for the equitypremium puzzle. This stems in part from restrictions that must be imposed on the functional forms of the utility and temptation specifications in order to properly take the model to the data. In particular, initial optimism was based on examples involving convex temptation functions, but 
convexity is inconsistent with balanced-growth behavior evident in the data. In replacing this specification with a concave temptation function that is consistent with balanced growth, the potential for self-control to help characterize the risk-free-rate and equity-premium puzzles becomes unclear a priori, and as we have found, is unfulfilled empirically. 


\section{References}

[1] DeJong, D.N., 1992. Co-Integration and Trend-Stationarity in Macroeconomic Time Series, Journal of Econometrics 52, 347-370.

[2] DeJong, D.N., B.F. Ingram and C.H. Whiteman, 2000. A Bayesian Approach to Dynamic Macroeconomics, Journal of Econometrics 98, 203-223.

[3] DeJong, D.N. and C.H. Whiteman, 1991. The Temporal Stability of Dividends and Stock Prices: Evidence from the Likelihood Function, American Economic Review 81(3), 600-617.

[4] DeJong, D.N. and C.H. Whiteman, 1994. Modeling Stock Prices Without Knowing How to Induce Stationarity, Econometric Theory 10, 701-719.

[5] Grossman, S.J. and R.J. Shiller, 1981. The Determinants of the Variability of Stock Market Prices, American Economic Review 71, 222-227.

[6] Gul, F. and W. Pesendorfer, 2004. Self-Control and the Theory of Consumption, Econometrica $72(1), 119-158$.

[7] Huang, K., Z. Liu, and Q. Zhu, 2005. Temptation and Self-Control: Some Evidence from the Consumer Expenditure Survey, Manuscript.

[8] Ingram, B.F., N.R. Kocherlatoka and N.E. Savin, 1994. Explaining Business Cycles: A Multiple Shock Approach, Journal of Monetary Economics 34, 415-428.

[9] Kocherlakota, N.R., 1996. The Equity Premium: It's Still a Puzzle. Journal of Economic Literature $34,42-71$.

[10] Krusell, P., B. Kuruscu and A.A. Smith, 2002. Time Orientation and Asset Prices, Journal of Monetary Economics 49(1),

[11] Lucas, R.J., 1978. Asset Prices in an Exchange Economy, Econometrica 46, 1429-1445.

[12] LeRoy, S.F. and R.D. Porter, 1981. Stock Price Volatility: Tests Based on Implied Variance Bounds, Econometrica 49, 97-113.

[13] Mehra, R. and E. Prescott, 2003. The Equity Premium in Retrospect, NBER Working Paper 9525. 
[14] Mehra, R. and E. Prescott, 1985. The Equity Premium: A Puzzle, Journal of Monetary Economics 15(2), 145-161.

[15] Shiller, R.J., 1981. Do Stock Prices Move Too Much to be Justified by Subsequent Changes in Dividends?, American Economic Review 71(3), 421-436.

[16] Weil, P., 1989. The Equity Premium Puzzle and the Risk-Free Rate Puzzle, Journal of Monetary Economics 24(2), 401-421. 
Table 1. Parameter Estimates

\begin{tabular}{|c|c|c|c|c|c|c|c|c|c|c|c|c|}
\hline & $\beta$ & $\gamma$ & $\lambda$ & $\rho_{d}$ & $\rho_{\mathrm{e}}$ & $\sigma_{\varepsilon \mathrm{d}}$ & $\sigma_{\varepsilon e}$ & $\operatorname{corr}\left(\varepsilon_{\mathrm{d}}, \varepsilon_{\mathrm{e}}\right)$ & $\sigma_{\text {up }}$ & $\sigma_{\text {ud }}$ & $\sigma_{\text {upf }}$ & $\sigma_{\mathrm{uc}}$ \\
\hline \multicolumn{13}{|l|}{ Mean } \\
\hline Prior & 0.960 & 2.000 & 0.0796 & 0.850 & 0.850 & UN & UN & UN & UN & UN & UN & UN \\
\hline CRRA & 0.995 & 3.546 & NA & 0.921 & 0.914 & 0.113 & 0.079 & 0.011 & $1.26 \mathrm{e}-6$ & $1.26 \mathrm{e}-6$ & 0.080 & 0.177 \\
\hline DSC & 0.995 & 3.524 & 0.0786 & 0.923 & 0.914 & 0.112 & 0.079 & 0.012 & $1.24 \mathrm{e}-6$ & $1.29 \mathrm{e}-6$ & 0.080 & 0.177 \\
\hline \multicolumn{13}{|l|}{ Std. Dev. } \\
\hline Prior & 0.020 & 1.000 & 0.060 & 0.025 & 0.025 & UN & UN & UN & UN & UN & UN & UN \\
\hline CRRA & $4.4 \mathrm{e}-5$ & 0.101 & NA & 0.022 & 0.010 & 0.007 & 0.006 & 0.085 & $3.66 \mathrm{e}-7$ & $3.69 \mathrm{e}-7$ & 0.006 & 0.012 \\
\hline DSC & $1.0 \mathrm{e}-4$ & 0.096 & 0.056 & 0.022 & 0.011 & 0.007 & 0.007 & 0.094 & 3.32e-7 & $3.96 \mathrm{e}-7$ & 0.012 & 0.056 \\
\hline
\end{tabular}

Notes: UN denotes “uninformative prior"; NA denotes "not applicable”. $\sigma_{\mathrm{ux}}$ denotes the standard deviation of the measurement error associated with the observation of variable $\mathrm{x}$. 


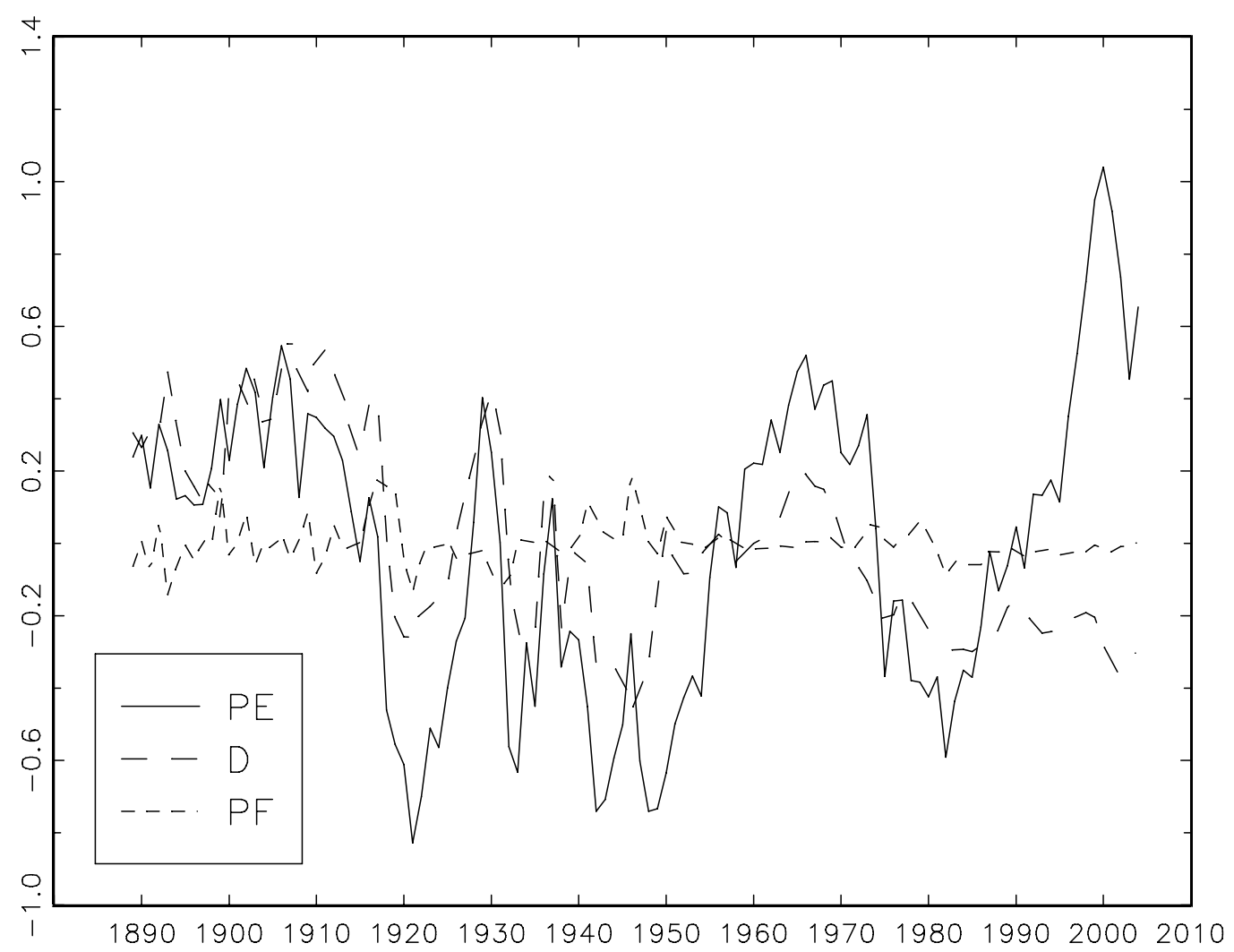

Figure 1. Asset Prices and Dividends (Logged Deviations from Trend) 
$\lambda$
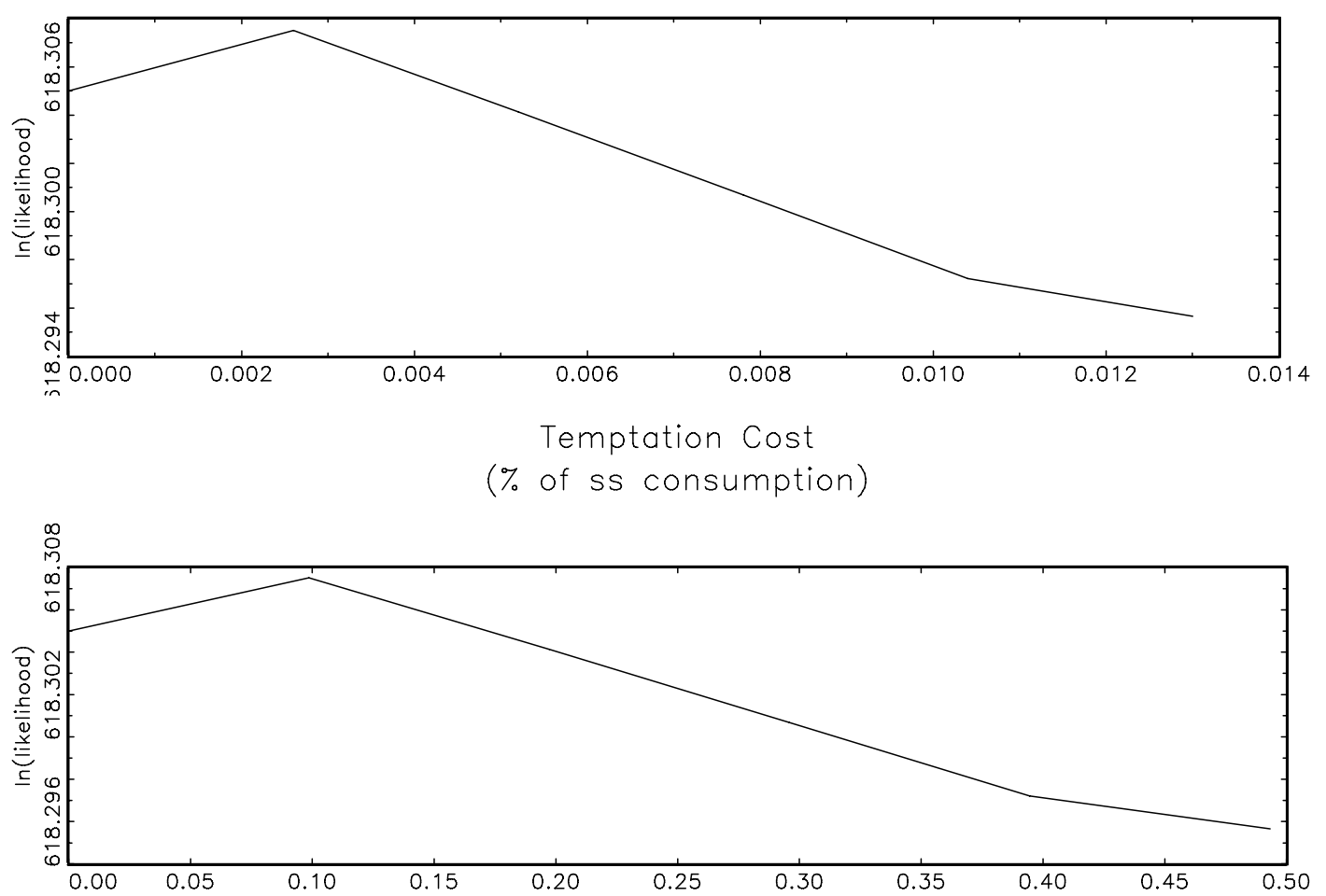

Figure 2. Conditional Likelihood Surface Over Temptation Parameter 
$\sigma(p) / \sigma(d)$

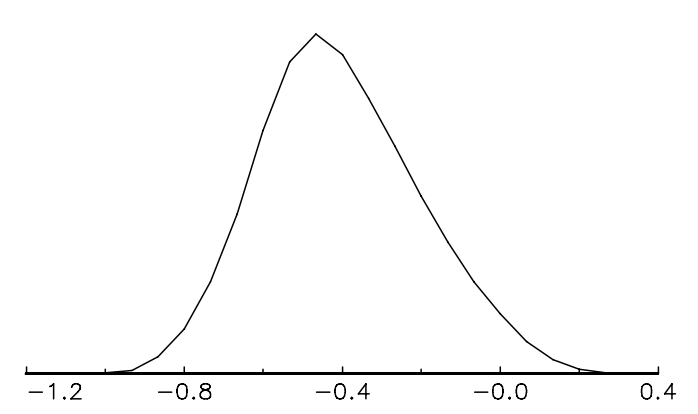

Equity Premium

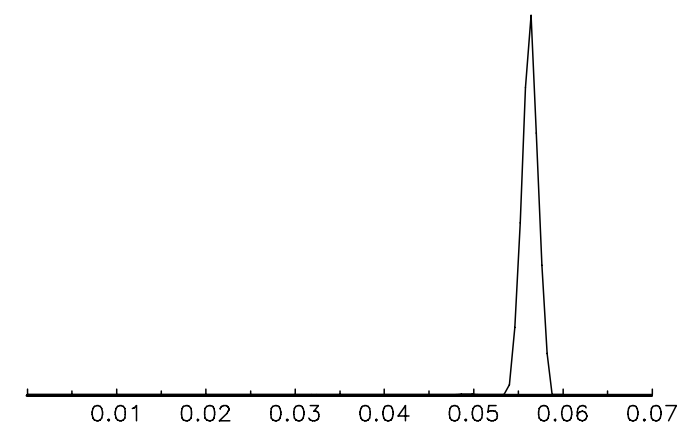

Corr(Price, Dividend)
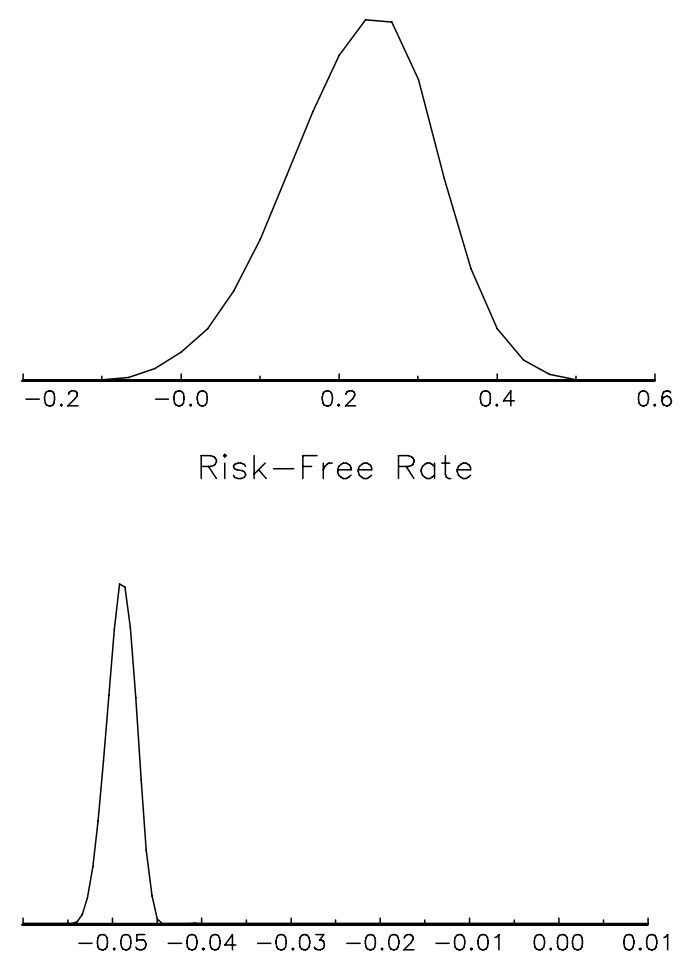

Figure 3. Summary Statistics, CRRA Preferences 
$\sigma(p) / \sigma(d)$

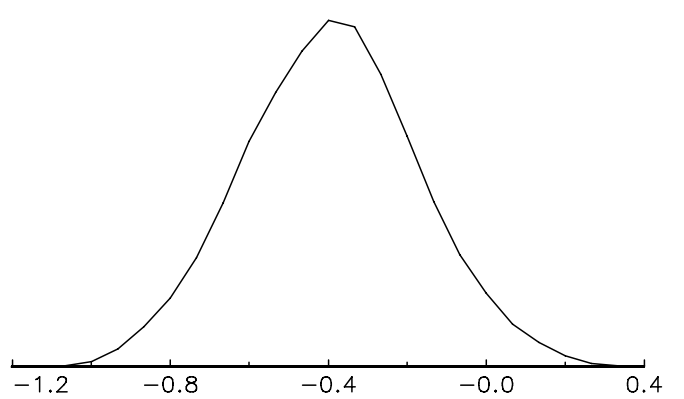

Equity Premium

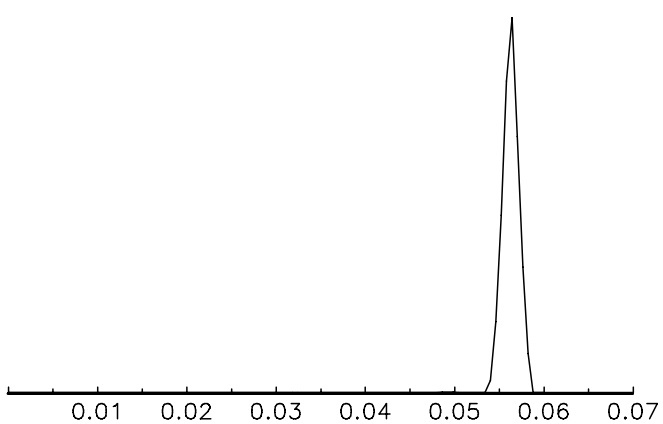

Corr(Price, Dividend)
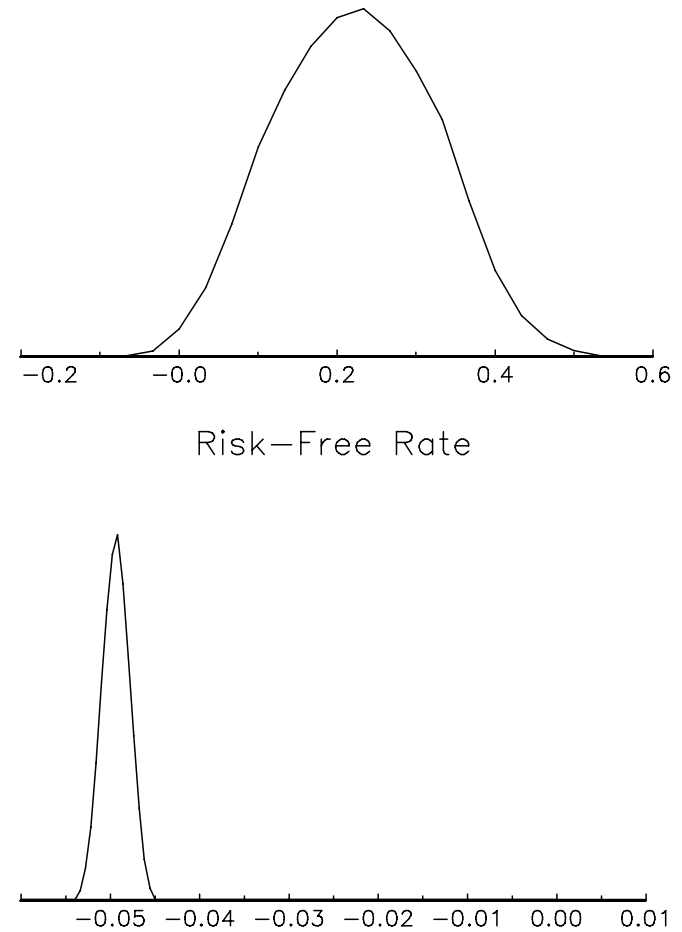

Figure 4. Summary Statistics, Temptation Preferences 